\title{
A CLINICAL AND ANALYTICAL STUDY OF CORRELATION BETWEEN DRY EYE SYNDROME AND REFRACTIVE ERROR IN YOUNG ADULT PATIENTS ATTENDING OPHTHALMOLOGY DEPARTMENT, MBS HOSPITAL KOTA.
}

\section{Dr. Jaishree singh}

\section{Dr. Parneet jat*}

\section{Dr. Äshok Meena}

Sr. Professor \& HOD Department of Ophthalmology, GMC KOTA, Rajasthan Pg Resident Doctor Department of Ophthalmology, GMC KOTA, Rajasthan ${ }^{*}$ Corresponding Author

\section{Sr. Professor Department of Ophthalmology, GMC KOTA, Rajasthan}

ABSTRACT Objectives:- This study aims to add new information about the DES in young adult by examining the correlation between dry eye syndrome and refractive errors.

Methods: In this cross sectional study, a total of 200 participants in the age range of 18 to 35 years and who were free of ocular surface disease, were taken from the patients attending outpatient Department of Ophthalmology, MBS Hospital Kota. Refraction was defined by the spherical equivalent (SE) as the following: 74 emmetropic eyes ( \pm 0.50 SE), 76 myopic eyes ( $\leq-0.75 \mathrm{SE}$ ), and 50 hyperopic eyes $(\geq+0.75 \mathrm{SE}$ ). All participants underwent full ophthalmic examinations assessing their refractive status and dryness level including tear film break up time, Rose Bengal staining and schirmer test.

Results: Prevalence of dry eye syndrome was $25.5 \%$ in this age group. In our study the prevalence of dry eye was $22.97 \%$, $31.57 \%$, and $20.00 \%$ in emmetropes, myopes, and hypermetropes, respectively ( $p$ value $=0.28$ ) and compared to emmetropes , prevalence of dry eye was higher in those with refractive errors (22.97\% and $26.98 \%$ respectively). Mean Tear breakup time in seconds showed shorter time in eyes of myopic and hyperopic individuals compared to emmetropia. Mean Rose Bengal staining score showed less in emmetropic individuals compared to myopic and hyperopic.

Conclusion: The current results succeeded to demonstrate a correlation between refractive errors and dryness level.

\section{KEYWORDS :}

\section{INTRODUCTION}

Dry eye syndrome (DES) is a common disorder that eye care providers encounter on a daily basis in which it accounts for $25 \%$ of patients' visits. ${ }^{1}$ Recently, the Tear Film and Ocular Surface Society DEWS II has revised the definition as follows:"Dry eye is a multifactorial disease of the ocular surface characterized by a loss of homeostasis of the tear film, and accompanied by ocular symptoms, in which tear film instability and hyperosmolarity, ocular surface inflammation and damage, and neurosensory abnormalities play etiological roles." ${ }^{2}$ Several risk factors have been reported to increase the risk of DES such as the is the long-term wear of contact lens and refractive surgery such as laser-assisted in situ keratomileusis or photorefractive keratectomy (LASIK). ${ }^{3.4}$ Questionnaire-based studies have shown that contact lens wearers have symptomatic DES than noncontact lens wearer.

A common finding between these studies is that approximately $50 \%$ of contact lens wearer reported dry eye. . $^{812}$ Interestingly, spectacle lens and contact lens wearers were twice and 12 times, respectively, more likely than emmetropes to report DES. ${ }^{13}$ Moreover, very few studies have shown evidence that refractive errors could contribute to the development of DES in young individuals.

\section{MATERIAL AND METHODS:}

Methods: In this cross sectional study, a total of 200 participants in the age range of $18-35$ years and who were free of ocular surface disease. This study was conducted on 200 patients in the Department of Ophthalmology, MBS Hospital Kota.

Refraction was defined by the spherical equivalent (SE) as the following: 74 emmetropic eyes ( $\pm 0.50 \mathrm{SE}), 76$ myopic eyes $(\leq-0.75 \mathrm{SE})$, and 50 hyperopic eyes $(\geq+0.75 \mathrm{SE})$. All participants underwent full ophthalmic examinations assessing their visual acuity by Snellen chart, refractive status (measurement of refractive error) using autorefractometer and subjective test, dryness level including (Fluorescein breakup time, Rose Bengal staining and schirmer test), slitlamp examination, Goldmann applanation tonometry, and the biomicroscopic fundoscopy (+90D lens) and assessment of meibomian gland, ocular surface disease index .
Diagnosis and confirmation of dry eyes will be done by series of tests performed in standard sequence as follows: Tear film breakup time (TBUT), ocular surface dye staining (Rose bengal stain), schirmer-1 test.

Diagnosis of dry eye in our study is made when two of the 3 tests are positive: TBUT, ocular surface dye staining and schirmer-ltest.

The study was approved by the Ethical Committee of govt medical college Kota and all patients recruited with informed consent.

\section{ELIGIBLE CRITERIA}

- Participants with best-corrected visual acuity of $6 / 9$

- Age range of 18-35 years

- Free of known ocular surface disease (Herpes simplex virus, varicella zoster virus, Steven Johnson Syndrome, aniridia, blepharitis etc.) are eligible for this study.

- Volunteer

\section{EXCLUSION CRITERIA}

Included those participants with

- ocular allergic disease

- keratitis

- contact lens wear

- glaucoma

- Ocular infection

- previous ocular surgery, eye lid surgery or injury

- Bell's palsy

- Systemic or ocular treatment.

- Unilateral Symptom

- Any Lid Pathology

- Meibomian gland dysfunction

- Smoker

\section{RESULTS:}

The age group was between 18 to 35 years, with the mean age of the patients being $25.54 \pm 5.89$ years. Among the entire group, 51 patients (87 eyes) were diagnosed to have dry eye based on the test, i.e. prevalence of $25.5 \%$.

Dry eye in relation to residence 
Out of 200 parents, 94 (47\%) were resident of urban area and $106(53 \%)$ were resident of rural areas. In our study prevalence of dry eye from rural areas were slightly higher than urban areas $26.41 \%$ and $24.46 \%$ respectively, Chi-square test was applied, $(\mathrm{p}$ value $=0.75$ )

\section{Dry eye in relation to sex}

Out of 200 parents, 96 were male and 104 were female. In our study prevalence of dry eye is slightly higher in female (26.92\%) than male (22.91\%), Chi-square test was applied, ( $p$ value $=0.51$ )

Dry eye prevalence in relation to refractive status

Prevalence of dry eye syndrome was $25.5 \%$ in this age group. In our study the prevalence of dry eye was $22.97 \%, 31.57 \%$, and $20.00 \%$ in emmetropes, myopes, and hypermetropes, respectively ( $p$ value $=0.28$ ) and compared to emmetropes , prevalence of dry eye was higher in those with refractive errors (22.97\% and $26.98 \%$ respectively).

Table no. 1 : Dry eye prevalence in relation to refractive status

\begin{tabular}{|c|c|c|c|}
\hline Refractive status & Dry eye present & Dry eye absent & Total \\
\hline Emmetropia & 17 & 57 & 74 \\
\hline Myopia & 24 & 52 & 76 \\
\hline Hypermetropia & 10 & 40 & 50 \\
\hline Total & 51 & 149 & 200 \\
\hline
\end{tabular}

Chi-square test was applied, p value $=0.28$

Graph no. 1 : Dry eye prevalence in relation to refractive status

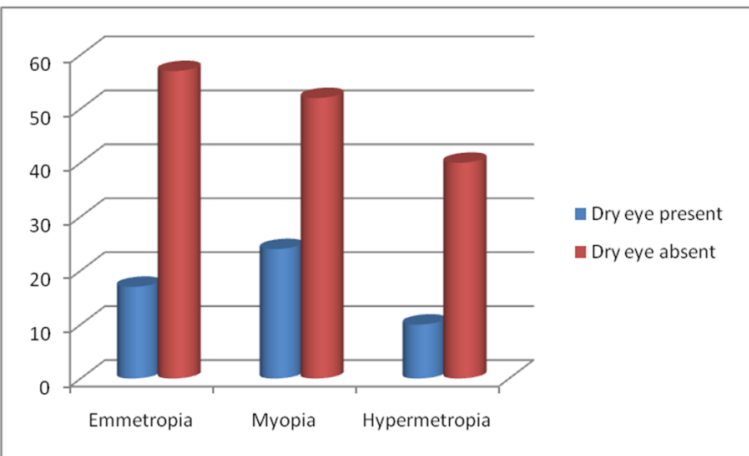

Table no.2: Dry eye prevalence in relation to emmetropia versus refractive error

\begin{tabular}{|c|c|c|c|}
\hline Refractive status & Dry eye present & Dry eye absent & Total \\
\hline Emmetropia & 17 & 57 & 74 \\
\hline Refractive error & 34 & 92 & 126 \\
\hline
\end{tabular}

Chi-square test was applied, $\mathrm{p}$ value $=0.52$

Graph no. 2 : Dry eye prevalence in relation to emmetropia versus refractive error

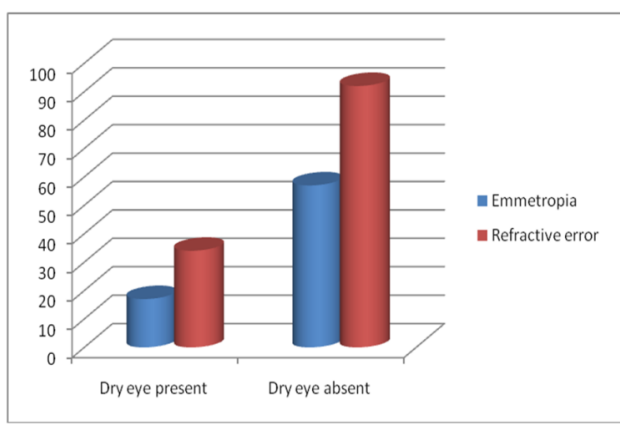

Mean value of TBUT in emmetropia, myopia and hypermetropia is 12.70, 11.79 and 12.36 Respectively. Mean Tear breakup time in seconds showed shorter time in eyes of myopic and hyperopic individuals compared to emmetropia.
Graph. no.3 : Mean value of TBUT

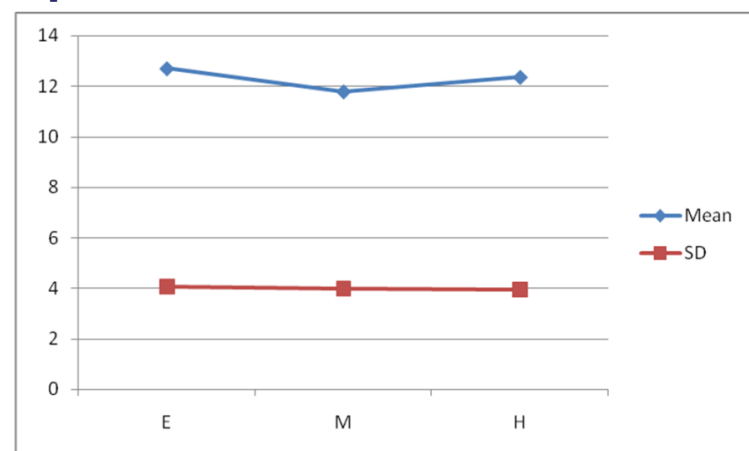

Mean value of Rose Bengal staining test in emmetropia, myopia and hypermetropia is 0.94, 1.14 and 1.02 Respectively. Mean Rose Bengal staining score showed less in emmetropic individuals compared to myopic and hyperopic.

\section{Graph no.4 : Mean value of Rose Bengal staining}

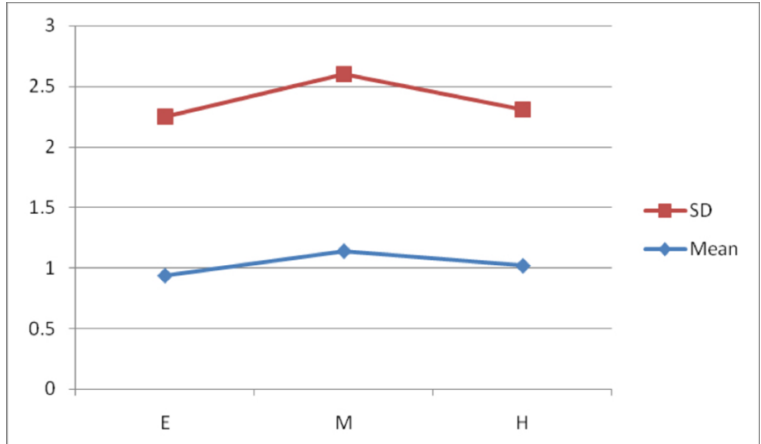

Mean value of Schirmer 1 test in emmetropia, myopia and hypermetropia is $16.71 \mathrm{~mm}, 16.67 \mathrm{~mm}$ and $16.77 \mathrm{~mm}$ Respectively.

Graph no.5 : Mean value of schirmer test

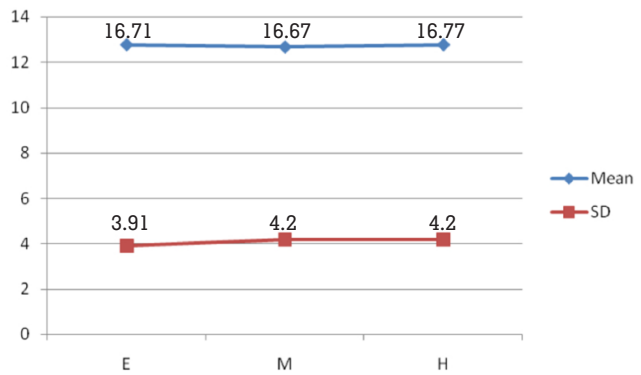

E- emmetropia, M- myopia, H- hyperopic

\section{DISCUSSION}

Dry eye is a distressing problem for both subjects and treating Ophthalmologist. The actual prevalence of this condition in the community is unknown because patients present for assessment and treatment when the condition is moderate to severe and the symptoms have become intolerable. Even at this stage, the diagnosis may not be made if the ophthalmologist does not perform the diagnostic tests required to detect dry eye. The condition of dry eye is therefore often overlooked and hence under diagnosed in the population. Dry eye is known to increase with age in both men and women, especially in post-menopausal females. ${ }^{14}$

The rate of dry eye in young adults is not well known although the visual tasks that younger adults are taking nowadays make them more vulnerable to DES. Video games, computers, and different digital devices as well as contact lenses are all contributing to the development of DES in younger patients. ${ }^{15}$ 


\begin{abstract}
${ }^{18}$ Studies have revealed that the overuse of smartphones is associated with increased risk of DES in children. ${ }^{19-21}$ Moreover, very few studies have shown evidence that refractive errors could contribute to the development of DES in young individuals. ${ }^{22,23}$
\end{abstract}

The aim of this study was to examine the prevalence of DES among young adults in relation to the refraction error.

This study was designed to find correlation between dry eye and refractive error in young adults in clinic setting, so the tests used were simple yet established in many studies to detect dry eye, namely the Schirmer's test, tear film break-up time and rose bengal dye staining. In our study, every patient had an ocular Examination for dry eye by the same investigator and this reduced bias and Standardised our diagnosis of dry eye.

The mean age of the group was $25.54 \pm 5.89$ years

\section{Prevalence of dry eye:}

In our study, out of 200, we found 51 patients to have evidence for dry eye either in one or both eyes based on the positive results of at least two out of three objective tests. The prevalence of dry eye in this study was found to be $25.5 \%$.

In a population based study in Indonesia, conducted by Lee $\mathrm{AJ}$ et $\mathrm{al}^{24}$ the prevalence of dry eye was $27.5 \%$.

The Salisbury ${ }^{25}$ eye study showed a prevalence of $14.6 \%$ based on subjects reporting symptoms.

The prevalence of dry eye varies from $10.8 \%$ to $57.1 \%$, there by showing wide disparity. ${ }^{26-30}$ The vast disparity in dry eye prevalence stems mainly from the different dry eye diagnostic criteria employed and different cut-off values for the objective dry eye tests. The high prevalence in some studies is also because objective dry eye tests have been performed in patients with positive symptom score ( thereby introducing a selection bias) or in patients in rheumatoid arthritis and Sjogren's syndrome, which have proven dry eye components. Our dry eye prevalence of $25.5 \%$ falls within this range.

Sex wise distribution of dry eye:

We found a slightly higher prevalence of dry eye $26.91 \%$ in women compared to $22.92 \%$ in men, which corresponded to the findings of other studies.

Moss et $\alpha^{31}$ found a prevalence of $16.7 \%$ in women compared to $11.4 \%$ in men. These were the prevalence rates obtained after adjusting for age.

Sahai et $\mathrm{al}^{32}$ found prevalence of $22.8 \%$ in women compared to $14.9 \%$ in men in his study on hospital based population.

\section{Residence wise distribution}

In our study prevalence of dry among patients from rural areas were slight higher than urban areas $(26.41 \%$ and $24.46 \%$ respectively). Though it was not statistically significant $(p=0.75)$. This incoherence with the study of Schuammberg et al $^{33}$ surveyed 39876 US women's health study, who reported there were no significant difference with respect to region of residence on the probability of having dry eye syndrome. In the study conducted by Sahai et $\mathrm{al}^{32}$ Dry eye appeared to be more common in rural (19.6\%) than urban patients $(17.5 \%)(\mathrm{P}=0.553 ; 95 \%$ CI 0.55-1.37); however this was not statistically significant.

\section{Refractive errors and dry eye:}

There are very few studies have shown evidence that refractive errors could contribute to the development of DES in young individuals.
In our study the prevalence of dry eye was $22.97 \%, 31.57 \%$, and $20.00 \%$ in emmetropes, myopes, and hypermetropes, respectively ( $p$ value $=0.28$ ) and compared to emmetropes , prevalence of dry eye was higher in those with refractive errors (22.97\% and 26.98\% respectively). Mean Tear breakup time in seconds showed shorter time in eyes of myopic and hyperopic individuals compared to emmetropia. Mean Rose Bengal staining score showed less in emmetropic individuals compared to myopic and hyperopic.

Our findings are consistent with other studies, Fahmy RM, Aldarwesh $\mathbf{A}^{34}$ Correlation between dry eye and refractive error in Saudi young adults using noninvasive Keratograph 4. The prevalence of dry eye was $24.6 \%, 36.5 \%$, and $17.4 \%$ in emmetropes, myopes, and hypermetropes, respectively. NIBUT has a negative correlation with hyperopia and a positive correlation with myopia with a significant reduction in the average NIBUT in myopes and hypermetropes in comparison to emmetropes.

Wang et al., ${ }^{35}$ who reported high prevalence of DES among myopic teenagers using Keratograph $5 \mathrm{M}$ and they demonstrated low tear volume in teenagers with myopia.

Unfortunately, the mechanism of refractive error inducing eye dryness is unknown. The cross sectional design of this study allowed screening of dryness among people with refractive error, but it would have been interesting to find the relation in terms of causality.

Nichols JJ et al. ${ }^{36}$ As stated earlier, individuals with refractive error are among those with a higher rate of contact lens and spectacle use as well as the reported rate of dryness.

Clinically, the changes in the anterior corneal surface as the eyeball elongates in myopia may contribute to increase the likelihood of developing dryness. ${ }^{37-39}$ In conclusion, noninvasive ocular surface examinations using Keratograph 4 showed a low NIBUT in healthy participants with refractive error which could be indicative of DES.

Sahai et $\mathrm{al}^{32}$ and Moss and colleagues..$^{31}$ which have shown that compared to emmetropes, prevalence of dry eye was higher in those with corrected and uncorrected refractive errors.

It has been postulated that persons with refractive errors have an increased tendency to rub their eyes which apart from introduction of infective material, sebum and sweat could cause the lodgement of particulate foreign substances into the eye that predispose to tear film instability. Also, people with uncorrected refractive errors have more tendency to squeeze the eye, causing instability of tear film, predisposing to dry eye. ${ }^{40}$

\section{CONCLUSION :}

Our conclusions from this study were as following:

1. Prevalence of dry eye syndrome was $25.5 \%$ in this age group.

2. There was no significant difference with respect to region of residence (urban vs rural) on the probability of having dry eye syndrome.

3. We found a slightly higher prevalence of dry eye in women (26.92\%) compared to men (22.91\%).

4. The prevalence of dry eye was $22.97 \%, 31.57 \%$, and $20.00 \%$ in emmetropes, myopes, and hypermetropes, respectively ( $p$ value $=0.28$ ). Prevalence of dry eye was higher in myopic as compared to other refractive status. Compared to emmetropes, prevalence of dry eye was higher in those with refractive errors $(22.97 \%$ and $26.98 \%$ respectively). 
The cross sectional design of this study allowed screening of dryness in relation to refractive error, but it would have been interesting to find the relation in term of causality. Hence more studies are required to understand the patho-physiology and prevalence of dry eye in relation to refractive status and should be treated effectively.

This study has shown evidence that refractive errors could contribute to the development of DES in young adults individuals.

\section{Financial support and sponsorship}

Nil.

\section{Conflicts of interest}

There are no conflicts of interest.

\section{REFERENCES}

1. O'Brien PD, Collum LM. Dry eye: Diagnosis and current treatment strategies. Curr Allergy Asthma Rep 2004;4:314-9.

2. The definition and classification of dry eye disease: Report of the definition and classification subcommittee of the international dry eye workShop (2007). Ocul Surf 2007:5:75-92.

3. Moss SE, Klein R, Klein BE. Prevalence of and risk factors for dry eye syndrome. Arch Ophthalmol 2000;118:1264-8.

4. Rapoport Y, Singer JM, Ling JD, Gregory A, Kohanim S. A comprehensive review of sex disparities in symptoms, pathophysiology, and epidemiology of dry eye syndrome. Semin Ophthalmol 2016;31:325-36.

5. Schaumberg DA, Sullivan DA, Buring JE, Dana MR. Prevalence of dry eye syndrome among US women. Am J Ophthalmol 2003;136:318-26.

6. Schaumberg DA, Sullivan DA, Dana MR. Epidemiology of dry eye syndrome. Adv Exp Med Biol 2002;506:989-98.

7. Craig JP, Nichols KK, Akpek EK, Caffery B, Dua HS, Joo CK, et al. TFOS DEWS II definition and classification report. Ocul Surf 2017;15:276-83.

8. Ang RT, Dartt DA, Tsubota K. Dry eye after refractive surgery. Curr Opin Ophthalmol 2001;12:318-22.

9. Donnenfeld ED, Ehrenhaus M, Solomon R, Mazurek J, Rozell JC, Perry HD, et al. Effect of hinge width on corneal sensation and dry eye after laser in situ keratomileusis. J Cataract Refract Surg 2004;30:790-7.

10. Begley CG, Chalmers RL, Mitchell GL, Nichols KK, Caffery B, Simpson T, et al. Characterization of ocular surface symptoms from optometric practices in North America. Cornea 2001;20:610-8.

11. Doughty MJ, Fonn D, Richter D, Simpson T, Caffery B, Gordon K, et al. A patient questionnaire approach to estimating the prevalence of dry eye symptoms in patients presenting to optometric practices across Canada. Optom Vis Sci 1997;74:624-31.

12. Guillon M, Cooper P, Maïssa C, Girard-Claudon K. Dry eye symptomatology of contact lens wearers and nonwearers. Adv Exp Med Biol 2002;506:945-9.

13. Brennan NA, Efron N. Symptomatology of HEMA contact lens wear. Optom Vis Sci 1989;66:834-8.

14. Schaumberg DA, Sullivan DA, Buring JE, Dana MR. Prevalence of dry eye syndrome among US women. Am J Ophthalmol 2003;136:318-26.

15. Uchino M, Schaumberg DA, Dogru M, Uchino Y, Fukagawa K, Shimmura S, et al. Prevalence of dry eye disease among Japanese visual display terminal users. Ophthalmology 2008;115:1982-8.

16. Kojima T, Ibrahim OM, Wakamatsu T, Tsuyama A, Ogawa J, Matsumoto Y, et al. The impact of contact lens wear and visual display terminal work on ocular surface and tear functions in office workers. Am J Ophthalmol 2011;152:933

17. Nichols JJ, Sinnott LT. Tear film, contact lens, and patient-related factors associated with contact lens-related dry eye. Invest Ophthalmol Vis Sci 2006;47:1319-28

18. Yokoi N, Uchino M, UchinoY, Dogru M, Kawashima M, KomuroA, et al. Importance of tear film instability in dry eye disease in office workers using visual display terminals: The Osaka study. Am J Ophthalmol 2015:159:748-54.

19. Moon JH, Lee MY, Moon NJ. Association between video display terminal use and dry eye disease in school children. J Pediatr Ophthalmol Strabismus 2014;51:87-92

20. Moon JH, Kim KW, Moon NJ. Smartphone use is a risk factor for pediatric dry eye disease according to region and age: A case control study. BMC Ophthalmol 2016;16:188.

21. Wagner RS. Smartphones, video display terminals, and dry eye disease in children. J Pediatr Ophthalmol Strabismus 2014;51:76

22. Ilhan N, Ilhan O, AyhanTuzcu E, Daglioglu MC, Coskun M, Parlakfikirer N, et al. Is there a relationship between pathologic myopia and dry eye syndrome? Cornea 2014;33:169-71.

23. Wang X, Lu X, Yang J, Wei R, Yang L, Zhao S, et al. Evaluation of dry eye and meibomian gland dysfunction in teenagers with myopia through noninvasive keratograph. J Ophthalmol 2016;2016:6761206.

24. Lee AJ, Lee J, Saw SM, Gizzard, Koh D, Widjaja D et al. Prevalence and risk factors associated with dry eye symptoms : A population based study in Indonesia. BrJ Ophthalmol 2002;86:1347-51.

25. Schein ODl, Muñoz B, Tielsch JM, Bandeen-Roche K, West S. Prevalence of dry eye among the elderly. Am J Ophthalmol. 1997 Dec;124(6):723-8.

26. Hikichi T, Yoshida A, Fukui Y, Hamano T, Ri M, Araki K, et al .Prevalence of dry eye in Japanese eye centres. Graefes Arch Clin Exp ophthalmol 1995 ; 233:555-8

27. Farrel J, Grierson DJ, Patel S, Sturrock RD . A classification for dry eyes following comparison of tear thining time with schirmer tear test. Acto Ophthalmol (Copenh) 1992; 70: 357-60.

28. Toda I , Fujishima H, Tsubota K. Ocular Fatigue is the major symptom of dry eye. Acta Ophthalmol (Copenh) 1993;71:347-52

29. Albietz JM. Prevalence of dry eye subtypes in clinical optometry practice Optometry Vis Sci 2000;77:357-63.

30. Versura P, Cellili M, Torreggiani A, Profazio V, Bernabini B, Caramazzo R. Dryness symptoms, diagnostic protocol and therapeutic management : A report on 1,200 patients. Ophthalmol Res $2001 ; 33: 221-7$.

31. MOSS SE, KLEIN R, KLEIN BE. Prevalence and risk factors for dry eye syndrome. Arch Ophthalmol 2000; 118: 1264-1268.

32. SAHAI A, MALIK P. Dry eye : prevalence and risk factor in hospital based population. Indian journal of Ophthalmology, 2005; 53: 87-91

33. Schaumberg DA, Sullivan DA, Buring JE, Dana MR. Prevalence of dry eye syndrome among US women. Am J Ophthalmol 2003;136:318-26.

34. Fahmy RM, Aldarwesh A. Correlation between dry eye And refractive error in Saudi young adults using noninvasive Keratograph 4.Indian J Ophthalmol 2018;66:653-6

35. Wang X, Lu X, Yang J, Wei R, Yang L, Zhao S, et al. Evaluation of dry eye and meibomian gland dysfunction in teenagers with myopia through noninvasive keratograph. JOphthalmol 2016;2016:6761206.

36. Nichols JJ, Sinnott LT. Tear film, contact lens, and patient-related factors associated with contact lens-related dry eye. Invest Ophthalmol Vis Sci 2006; $47: 1319-28$

37. Jie Y, Xu L, Wu YY, Jonas JB. Prevalence of dry eye among adult Chinese in the Beijing eye study. Eye (Lond) 2009;23:688-93.

38. Xu L, Wang YX, Guo Y, You QS, Jonas JB, Beijing Eye Study Group. Prevalence and associations of steep cornea/keratoconus in greater Beijing. The Beijing eye study. PLoS One 2012;7:e39313.

39. Chang SW, Tsai IL, Hu FR, Lin LL, Shih YF. The cornea in young myopic adults BrJOphthalmol 2001;85:916-20.

40. HIKICHI T, YOSHIDA A, FULKI Y, HAMANO T, RI M, ARAKI K. Prevalence of dry eye in Japanese eye centres. Graefes Arch Clin Exp O phthalmol 1995; 233 555-558. 